\title{
COMPARISON WITH ACCURACY OF TERRESTRIAL LASER SCANNER BY USING POINT CLOUD ALIGNED WITH SHAPE MATCHING AND BEST FITTING METHODS
}

\author{
T. Ogawa ${ }^{1}$, Y. Hori ${ }^{2}$ \\ ${ }^{1}$ Faculty of Human-Environment Studies, Kyushu University, 744, Motoka,Fukuoka 812-8581- ogawa.takuro.759@ s.kyushu-u.ac.jp \\ ${ }^{2}$ Dept. of Architecture and Urban Design, Kyushu University, 744, Motoka, Fukuoka 812-8581 - hori@arch.kyushu-u.ac.jp
}

\section{Commission II}

KEY WORDS: Laser scanning, time of flight, phase shift, shape matching, natural distributions, Ostia

\begin{abstract}
:
Recently operation systems of laser scanning have been obviously improved; for instance shape matching has been equipped with software on a post processing stage so measurement without any targets is a prerequisite condition of field surveying with laser scanners. Moreover a shape matching method enables us to easily register a pair of point clouds with some errors even if those data are scanned by several type scanners. Those slightly errors can influence accuracy of alignments if the object is large to require a lot of scans. Laser scanning data has random errors and accuracy of alignments can be improved by matching error distributions of pairs of point clouds to natural distributions. This method is called "best fitting" in contrast "shape matching" in a software, PolyWorks |Inspector. In this paper, accuracy of alignments between shape matching and best fitting is discussed. The scan data of three phaseshift laser scanners (FARO Focus 3D MS120, FARO Focus 3D X330 and Z+F Imager 5016) and two time-of-flight scanners (Leica BLK 360 and Leica Scan station C5) are used for analyses. Accuracy of alignments by using shape matching and best fitting methods is demonstrated by showing points of scan data with histograms of error distributions.
\end{abstract}

\section{INTRODUCTION}

Remote sensing technology has been adopted into field survey and a lot of researchers have reaped a rich harvest from it for decades. Recently operation systems of laser scanning have been obviously improved; for instance shape matching has been equipped with software on a post processing stage so measurement without any targets is a prerequisite condition of field surveying with laser scanners. Moreover commercial software enables us to align pairs of overlapping scans in error of a few millimetres in average, even if those pairs are measured by several kinds of laser scanners in different time and situations.

While aligning point clouds by a shape matching method, hundreds to thousands points are usually picked up at random and software calculates to match their positions with less errors. This operation is repeated until average errors between pairs of point clouds fulfil a set parameter. However, point cloud data has random errors and in shape matching process, distributions of errors between pairs of point clouds after shape matching are usually not matched to natural distributions (the graph on the left side on figure 1). This graph shows that a histogram of distributions of errors after shape matching usually has two peaks and it is not along natural distributions. That is, the data of pairs of point clouds, which shows such a histogram, could be aligned better until the histogram becomes to be like illustrated on the graph on the right side on figure1, which matches almost natural distributions.

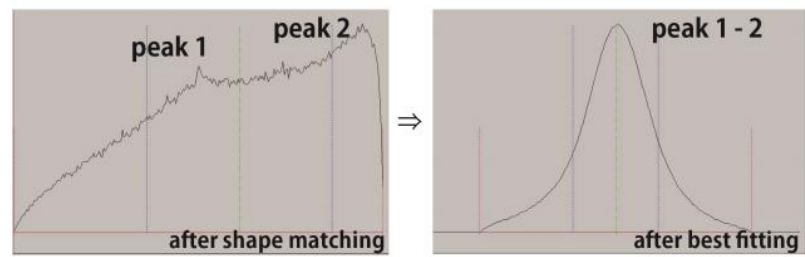

Figure 1: The Example of Histgrams of error distributions (PolyWorks IMAlign was used.)
The distributions of random errors among point cloud after best fitting alignment tend to be ignored because such difference is not oblivious in many cases. However the larger scale objects are and the more number of scans increase, the more unavoidable difference between scans will be made. Those errors can be kept to a minimum by calculating with a least squares method and approximating their histograms to ideal shapes of natural distribution (This method is usually called "best fitting" in contrast "shape matching" in a software, PolyWorks |Inspector).

Best fitting alignment is appropriate to measure specific objects which require several kinds of laser scanners and a lot of time due to their scale and shapes. Japanese team has carried out laser scanning the whole of ancient Roman city, Ostia in Italy from 2012. This project required a lot of time so laser scanning has been carried out with several times updating laser scanners in order to be done more efficiently. Now some objects have been scanned by maximum five types of laser scanners and each data is aligned with shape matching and best fitting methods.

In this paper, accuracy of alignment data, which contains two time-of-flight terrestrial laser scanners (Leica Scanstation C5 and Leica BLK360) and three phase-shift terrestrial laser scanners (FARO FOCUS 3D MS120, FARO FOCUS 3D X330 and $\mathrm{Z}+\mathrm{F}$ Imager 5016), will be demonstrated by using shape matching alignment and best fitting alignment.

\section{BACKGROUND AND SCAN DATA INFORMATION}

In previous researches the accuracy behaviours of some kinds of terrestrial laser scanners were investigated, (Klaus et al, 2007). For instance of employment of the practice by using two types of laser scanners and best fitting alignment, the accuracy of time-of-flight and phase-shift laser scanners were also compared in the same situation while scanning objects, (San José Alonso et al, 2011). Those researches focus on the performances and precisions of each laser scanner whereas we discuss about how we can gain more accurate results of measuring by using different scanners. Up-to-date laser scanners can measure objects more accurately than before so the 
differences of performances have been narrowed. Moreover laser scanning technologies are advancing while being subdivided so scanners can be selected adopting various kinds of objects and situations. It is not special that one team uses point clouds of different kinds of laser scanners. Both of shape matching and best fitting methods usually use a pair of point clouds as a reference scan and a moving scan even if objects are measured by several kinds of scanners.

This paper is constructed by two analyses, in which parts of the wall surfaces of the ancient roman temple are used as an object. Firstly accuracy in both of shape matching and best fitting is examined by comparing alignments of pairs of point clouds which were measured by the same scanners. Leica BLK360 and $\mathrm{Z}+\mathrm{F}$ Imager 5016 are used in this demonstration. Secondly accuracy of alignments of five scans, which are measured by each scanner (FARO Focus 3D MS120, FARO Focus 3D X330, Leica BLK360, Leica Scanstation C5 and Z+F Imager 5016) and aligned by shape matching and best fitting methods, are compared with each other. Then it is inspected whether common alignments of points of several scanners, exist or not. The object is the south-west wall of the temple in Ostia, the ancient Roman city which is located at the mouth of Tiber in Italy. The temple is called the Capitolium and this wall is approximately $17 \mathrm{~m}$ height and $19 \mathrm{~m}$ wide. It is the appropriate object out of the buildings which exist in the whole of the city to be examined the difference of alignment with shape matching and best fitting because everywhere conditions on large surface of the wall are not so changed.

For the examinations, seven point clouds were used without changing any part and the information of each point cloud data is written down below (fig.2). Each range of scans from scanners to an object is up to approximately $30 \mathrm{~m}$ and performance of each scanner is kept within its ranging accuracy. Each scan was taken in the different situations and settings. Scan data is used without removing and masking any points because the wall lacks characteristic shapes and it supposes to fail in aligning without information of shapes around the wall.

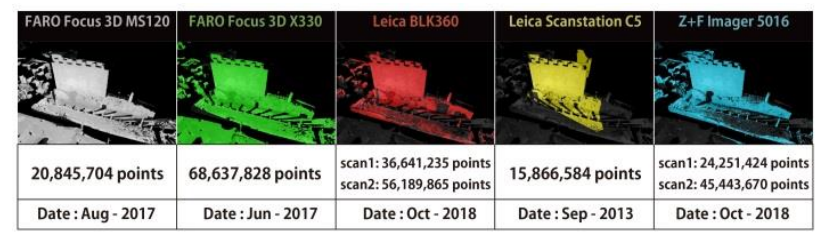

Figure 2: Information of Each Point Cloud

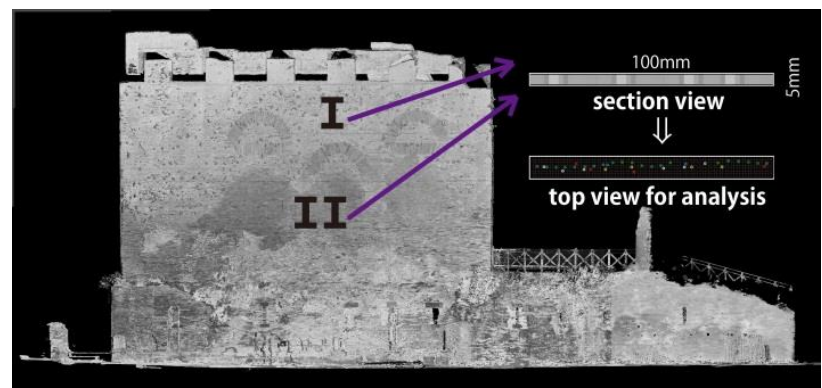

Figure 3: The Sample Objects Picked up for the Demonstration

Uneven surfaces of the west-side wall of the temple were cut out as two squares (squares I and II in figure 3) almost randomly. Those squares were so small $(100 \mathrm{~mm}$ wide and $5 \mathrm{~mm}$ height) that we can ignore shapes more than a few centimetres. Results of demonstrations can be also accepted even if any parts of the wall are selected because systematic and random errors rise without remarkable bias when laser scanning is carried out under appropriate situations and operations. Figure 3 illustrates the positions of squares which were analysed. Each square is shown in top view and $1 \mathrm{~mm}$ square grids are drawn in figures (fig. 5, 7, 9, 11, 13, 15 and 16).

\section{THE DIFFERENCE OF SHAPE MATCHING ALIGNMENT AND BEST FITTING METHODS}

The difference between shape matching and best fitting methods would be whether those methods match distributions of errors to normal distributions or not. Laser scanning usually has systematic errors and random errors. A distribution of random errors is assumed to be along normal distributions. In a shape matching method, an average of error distributions between points of scans is usually calculated and software has own methods. However those methods are not disclosed.

Shape matching alignment is a method to align one scan with another scan. This method is finished when average errors fall below the tolerance, for instance less than $1 \mathrm{~mm}$. Average error is calculated by distance between pairs of point clouds which are randomly selected out of scans. Points of pairs must overlap and more than $60 \%$ overlapping is appropriate for shape matching.

Best fitting alignment is the method to match a point cloud to the other one more accurately after shape matching alignment. This method is finished when the average error becomes kept to a minimum and distributions of errors are along normal distribution. Best fitting does not work well when scans have considerable errors such as compensator out due to winds.

\section{COMPARISON WITH SHAPE MATCHING ALIGNMENT AND BEST FITTING ALIGNMENT BY USING THE SAME SCANNER'S DATA}

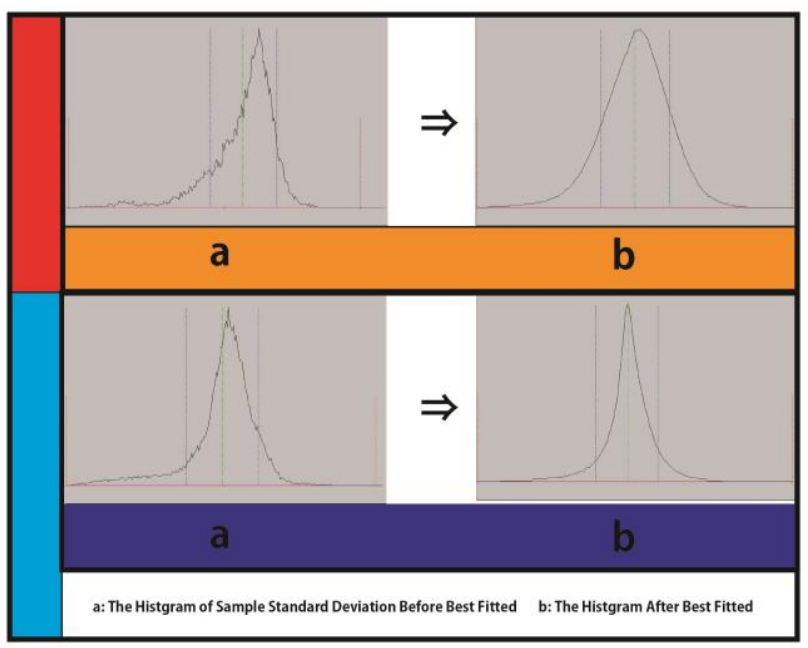

Figure 4: The Histgrams of Leica BLK360 and Z+F Imager 5016 (IMAlign was used.)

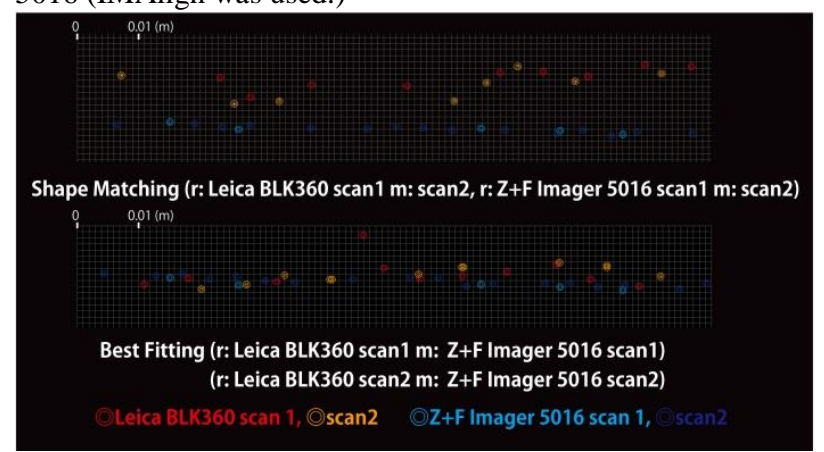

Figure 5: Alignments of Point Clouds of Leica BLK360 and $\mathrm{Z}+\mathrm{F}$ Imager 5016 
Both of Z+F Imager 5016 and Leica BLK360 have shape matching process within their software. Points of Leica BLK360 are coloured in red and points of $\mathrm{Z}+\mathrm{F}$ are coloured in blue. Both of scan 1 and 2 in each scanner were used for demonstrations. Scan 1 was a reference scan and scan 2 was a moving scan while aligning. Figure 4 illustrates lines of points aligned by using their shape matching methods install on their operational software. In figure 4 , blue points improve more accuracy than red ones and blue points have errors of within approximately $1 \mathrm{~mm}$ in depth direction. On the other hand, red points have errors of 1-7 $\mathrm{mm}$ in the same direction of blue points after shape matching. When those point clouds are aligned by best fitting method, firstly blue scan 1 was reference and scan 2 was matched to blue scan 1 . Secondly blue scan 1 and 2 were reference points for both of red scan 1 and 2. After best fitted, moving scans slightly slid and outlines of uneven surfaces of the wall became more clear (fig.5). Some red points, which are apart from those out lines, could be noise. As shown in figure 5, especially red points vary obviously after shape matching. Those red points are matched to blue points and reduced their difference of alignments.

\section{COMPARISON WITH SHAPE MATCHING ALIGNMENT AND BEST FITTED ALIGNMENT BY USING THE DIFFERENT SCANNER'S DATA}

Both of a shape matching method and a best fitting method use pairs of scans; one is reference scan and the other one is moving scan. Out of five different scans can be reference scans so all combinations were examined. The results will be written below in each reference scan. Figure 6, 8, 10, 12 and 14 illustrate three results of aligning: average errors by shape matching, error distributions before best fitting and after best fitting. Histograms in this figure are distributions of average errors of a pair of two scans picked up. Those histograms become more similar to natural distributions, results of aligning are more accurate. Figure 7, 9, 11, 13 and 15 show distributions of points in $1 \mathrm{~mm}$ grids by shape matching and best fitting in part I and II. Points of five scanners are distinguished by colours.

All scans were aligned by best fitting with tolerance in maximum $20 \mathrm{~mm}$. All scanners name are abbreviated such as MS120, X330, BLK360, C5 and Z+F below. Sizes of some images in fig $7,9,11,13,15$ and 16 were slightly different because rendering settings are changed due to a process to export images. However the differences in width of $1 \mathrm{~cm}$ among images do not change results of analysing and it could be ignored.

\subsection{FARO Focus 3D MS120 as the Reference Scan}

Every scan is aligned keeping average errors less than $3 \mathrm{~mm}$ after shape matching (fig.6). On the other hand, the histograms after shape matching are not along natural distributions. The histograms by best fitting except $\mathrm{C} 5$ would not declared that they are along natural distribution. In part I, distributions of points are not in order after best fitted compared with those of before best fitted (fig.7). In part II, points of BLK360 and C5 after shape matching is approximately $2-3 \mathrm{~mm}$ away from the other points. After best fitting, C5, several points of BLK360 and MS120 are also approximately $2-3 \mathrm{~mm}$ away from points of other scans. Best fitting alignment did not work well because scan data of MS120 would have remarkable errors.

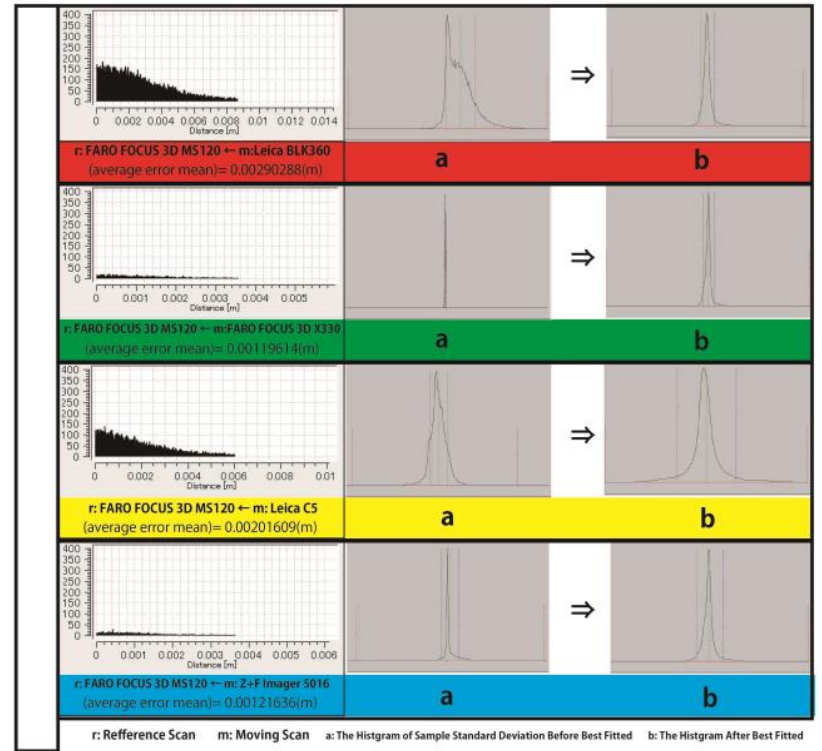

Figure 6: The Results of Alignments (Refference:FARO Focus 3D MS120), (JRC 3D Recnstructor and IMAlign were used)

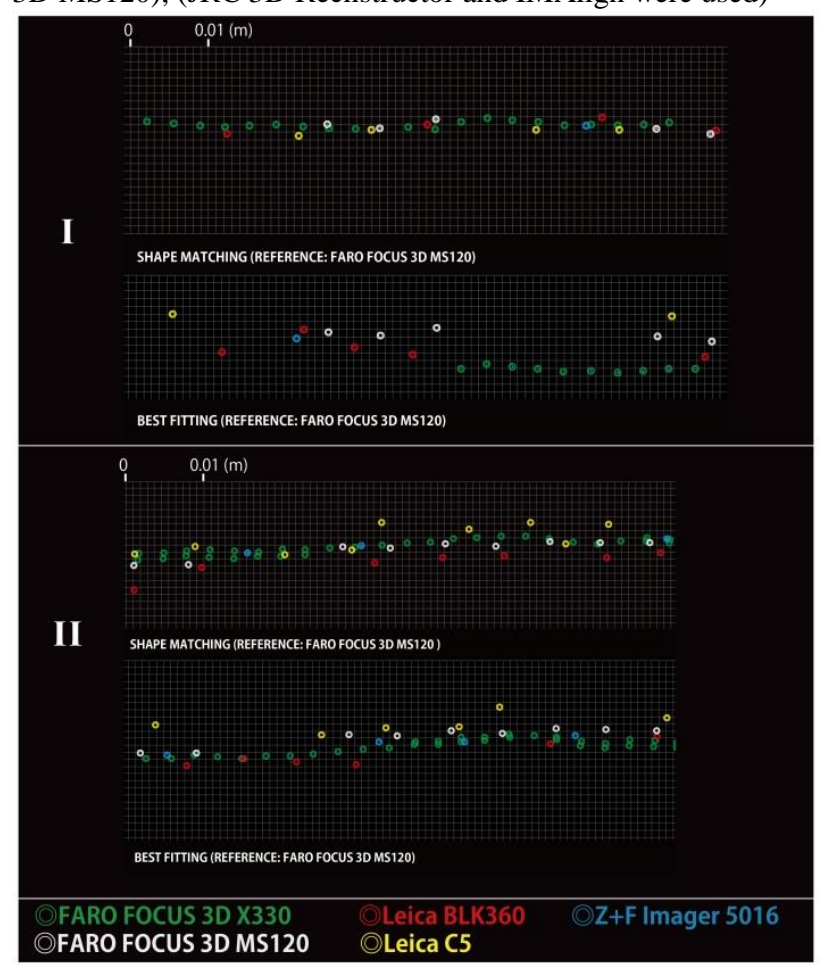

Figure 7: Alignments of Point Clouds (Refference:FARO Focus 3D MS120)

\subsection{FARO Focus 3D X330 as the Reference Scan}

Points of $\mathrm{Z}+\mathrm{F}$ are aligned with those of X330 in average errors less than $9 \mathrm{~mm}$ by shape matching (fig.8). The other points of scans are aligned keeping average errors less than $3 \mathrm{~mm}$. The histograms of the moving scans $\mathrm{C} 5$ and $\mathrm{Z}+\mathrm{F}$ are similar to natural distributions. In part I, positions of points after shape matching and after best fitting are not so different (fig.9). In part II, points of BLK360 and several points of C5 are approximately $2-3 \mathrm{~mm}$ away from the other scans after shape matching. Several points of MS120 are also slightly away less than those of BLK360 and C5. On the other hand, only points of BLK360 kept 2-3mm distance from the other points and the peak of the histogram of BLK360 does not match that of natural distribution. 


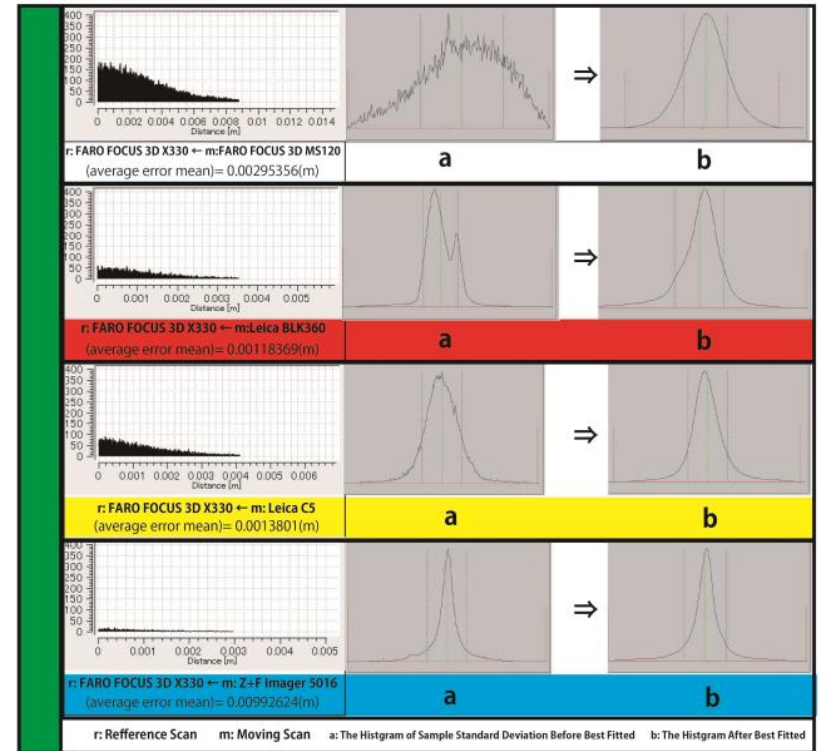

Figure 8: The Results of Aligning (Refference:FARO Focus 3D X330), (3D Recnstructor and IMAlign were used)

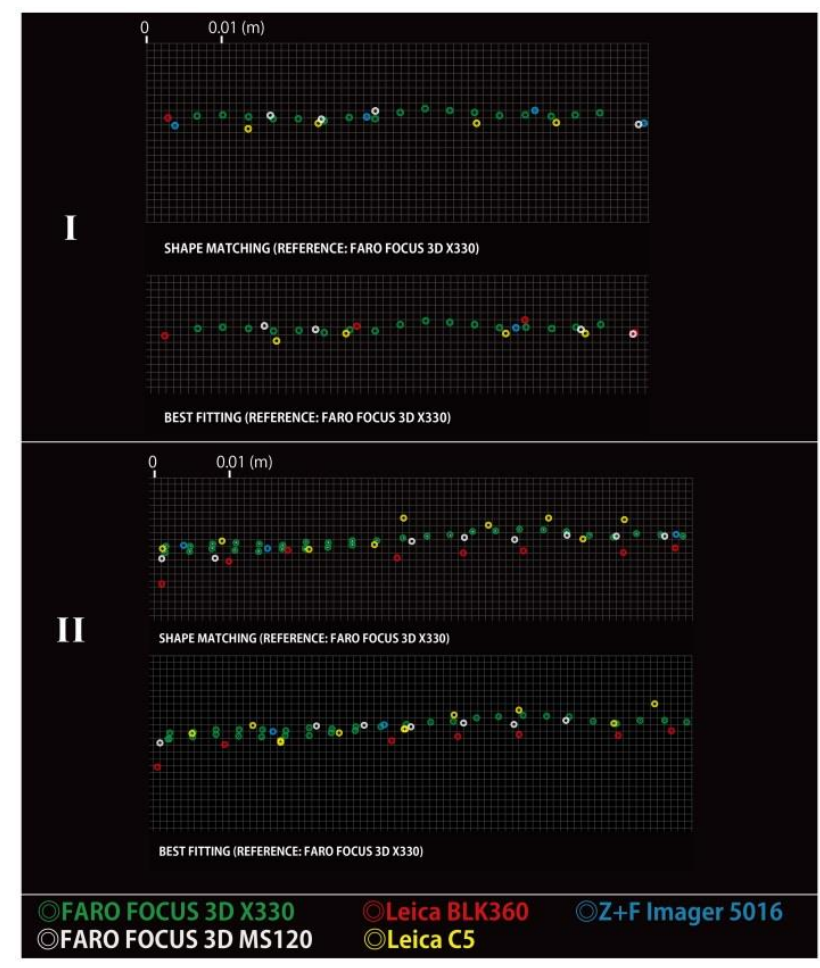

Figure 9: Alignments of Point Clouds (Refference:FARO Focus 3D X330)

\subsection{Leica Scan station C5 as the Reference Scan}

Average errors are kept less than $4 \mathrm{~mm}$ but peaks of histograms of all scanners after best fitting do not match slightly the centre of natural distribution (fig.10). In shape matching, points look like aligning orderly in part I whereas points of BLK360 and several points of $\mathrm{C} 5$ are approximately $2-5 \mathrm{~mm}$ away from the other points (fig.11). After best fitted, points of all scanners are aligned in few errors in part I. Points except several points of BLK360 and C5 are also aligned orderly in part II.

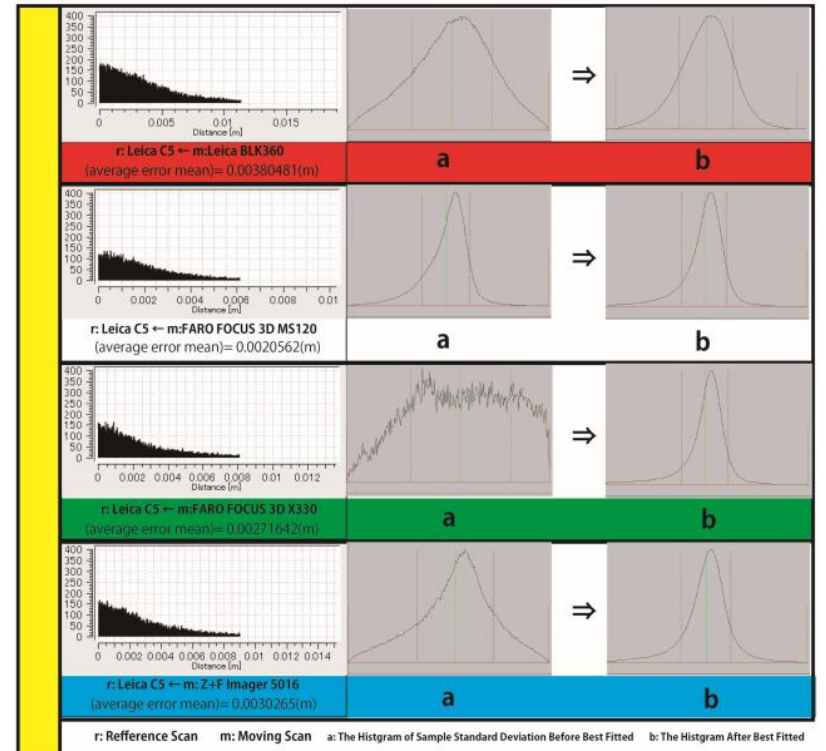

Figure 10: The Results of Aligning (Refference:Leica Scan Station C5), (3D Recnstructor and IMAlign were used)

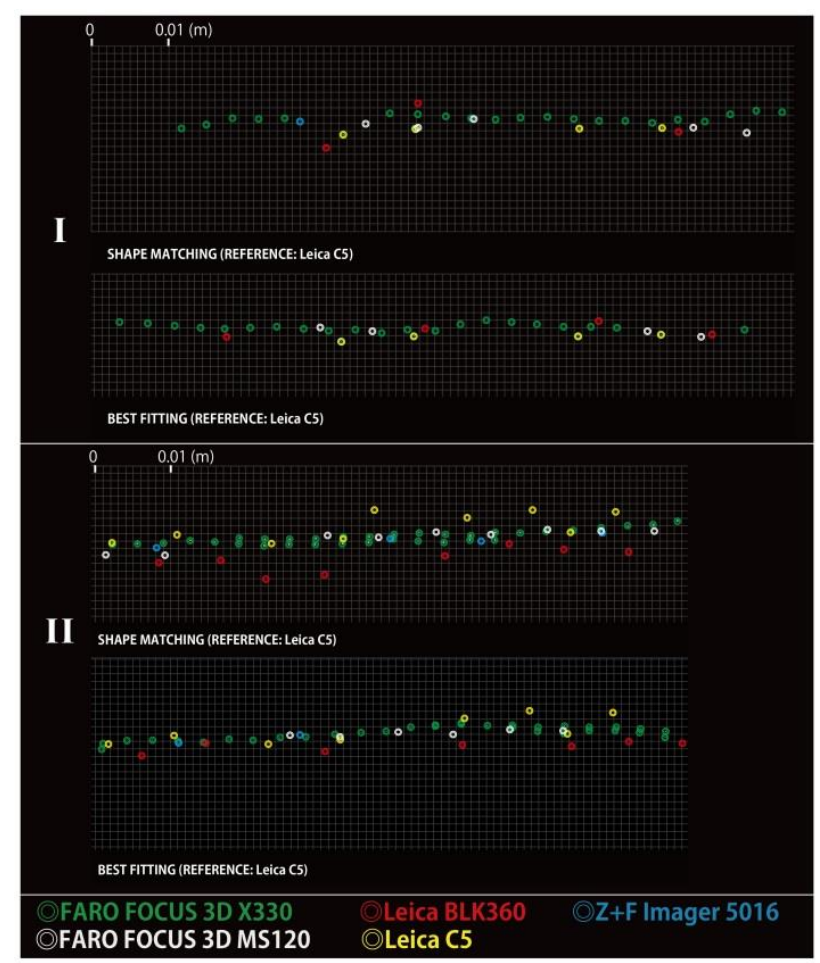

Figure 11: Alignments of Point Clouds (Refference:Leica Scan Station C5)

\subsection{Leica BLK360 as the Reference Scan}

All points are aligned keeping average errors to around $3-4 \mathrm{~mm}$ after shape matching and histograms except points of $\mathrm{Z}+\mathrm{F}$ match that of natural distribution after best fitting (fig.12). A peak of the histogram of $\mathrm{Z}+\mathrm{F}$ does not much the centre. After best fitted, almost all points are aligned orderly in part I (fig.13). On the other hand, points of BLK360 and several points of MS120 do not slightly match to lines of the other points in part II. 


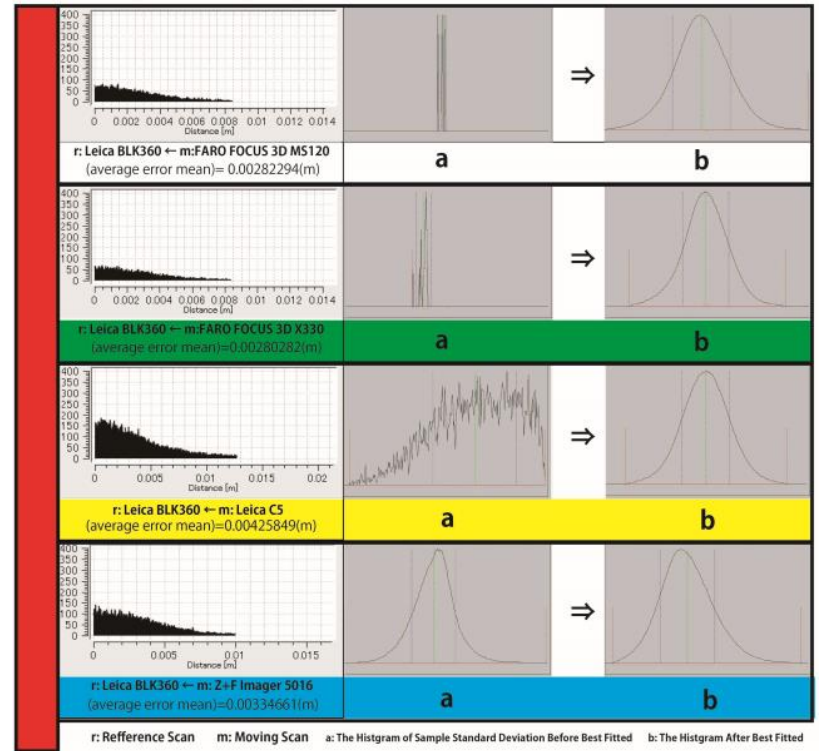

Figure 12: The Results of Aligning (Refference:Leica BLK360), (3D Recnstructor and IMAlign were used)

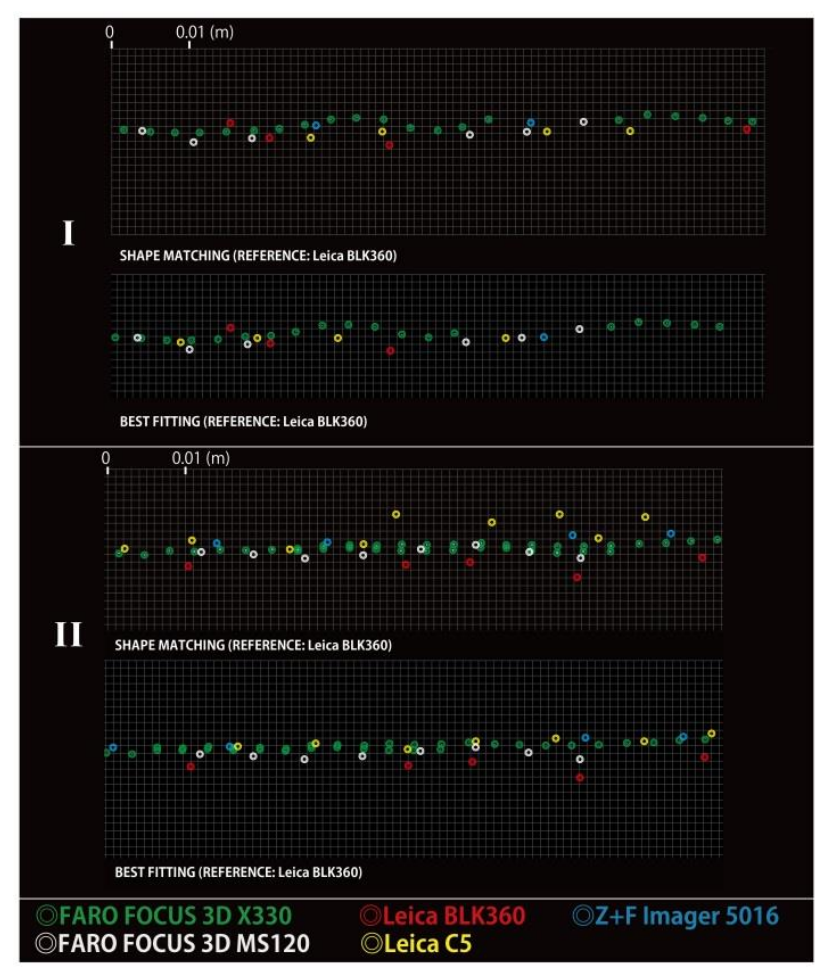

Figure 13: Alignments of Point Clouds (Refference:Leica BLK360)

\subsection{Z+F Imager 5016 as the Reference Scan}

All points are aligned in average errors within approximately $3 \mathrm{~mm}$ after shape matching and all histograms of scan data are along with natural distribution after best fitting (fig.14). Several points of MS120, BLK360 and C5 are kept errors to 1-2mm after best fitted in part I and II. Especially points of BLK are approximately $2-5 \mathrm{~mm}$ away from the other points in part II (fig.15).

\subsection{Evaluation the demonstrations}

Some common distributions of points, which are mixed points of several scanners, exist on figure $9,11,13$ and 15 . Those

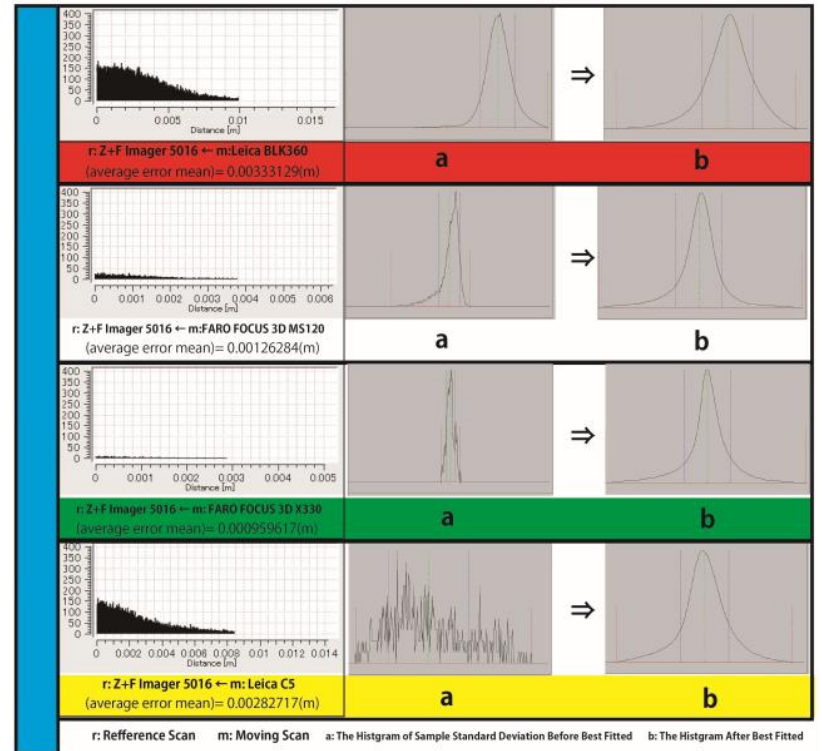

Figure 14: The Results of Aligning (Refference:Z+F Imager 5016), (3D Recnstructor and IMAlign were used)

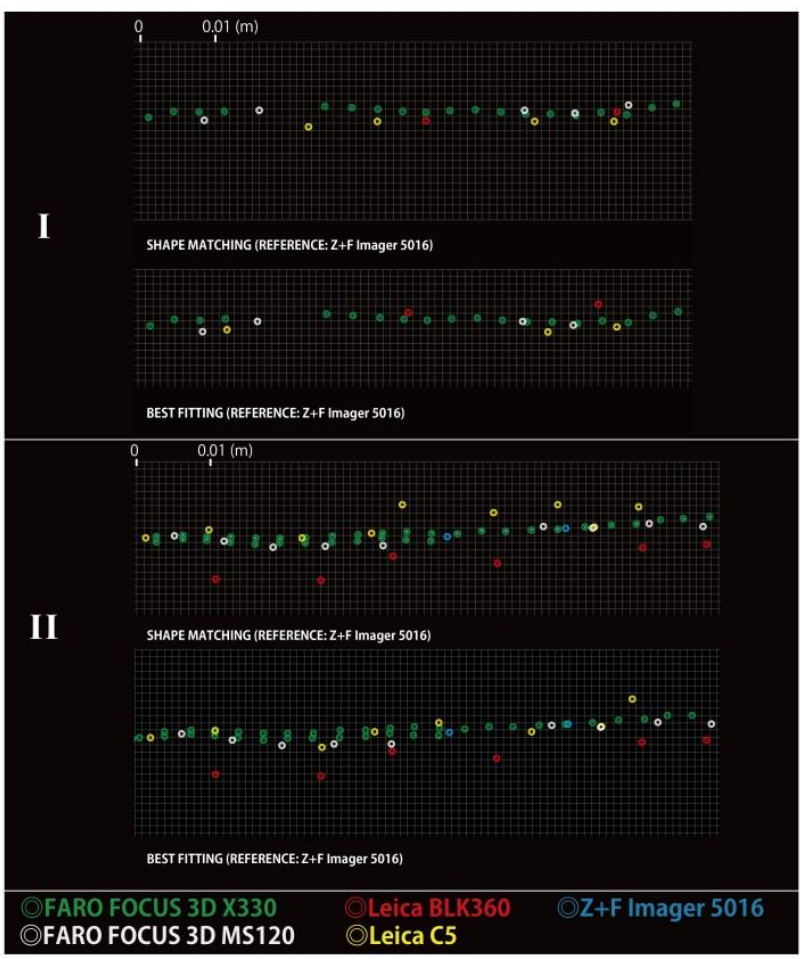

Figure 15: Alignments of Point Clouds (Refference:Z+F Imager 5016)

distributions are assumed to be more accurate data because almost the same distributions are gained in some alignments, in which point clouds of different laser scanners are reference scans. On the other hand, several points not along with those common distributions would be random errors. An example of those distributions is picked up in figure 16. As a result of analysing, those common lines can be seen after best fitting in the data in which reference scans are point clouds of Leica C5, FARO X330 and Z+F Imager 5016. Leica BLK360 has parts of those common lines. After shape matching it could be considered that moving scans slightly slide on the surface of the reference scan, even if average errors are kept to a few millimetres and it looks almost no errors on alignments of 
points in the depth direction. A shape matching method keeps errors within range from a few centimetres to millimetres in reality according to comparison with alignments of each point with a best fitting method. On the other hand, a best fitting method keeps errors within a few millimetres as a result illustrated with figure 16. Furthermore the common distributions are gained on keeping errors within a millimetre. The results also vary between time-of-flight and phase-shift laser scanners. In shape matching, points of square I are aligned less error in depth direction than those of square II. That is supposed shape matching method use characteristic shapes such corners as markers and square $I$ is closer to the corners than square II is. In shape matching, points of time-of-flight laser scanners and phase-shift laser scanners tend not to match each other. After best fitting, points of both of those scanners are aligned together and accuracy of alignments would depend on precisions and random errors of each laser scanner.

Point clouds which were used for analysing were scanned in different situations and settings and this paper does not discuss about precisions of each laser scanner. We focus on accuracy of alignments of point clouds by using different kind of scanners and methods. The proper number of points of the data for accurate alignment could not be referred to in those demonstrations.

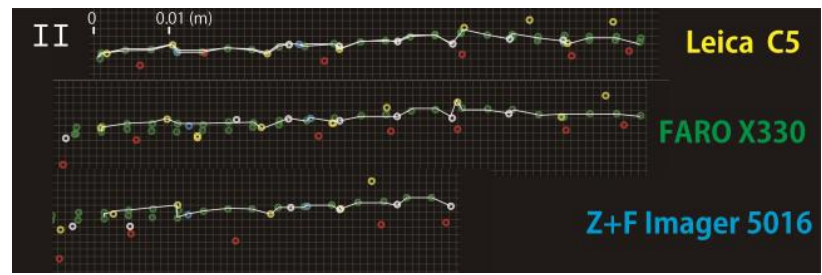

Figure 16: An example of common lines in Leica C5, FARO $\mathrm{X} 330$ and $\mathrm{Z}+\mathrm{F}$ in partII

\section{CONCLUSION}

Shape matching has been equipped with software on a post processing stage so measurement without any targets is a prerequisite condition of field surveying with laser scanners. Moreover a shape matching method enables us to easily register a pair of point clouds with some errors even if those data are scanned by several type scanners. Those slightly errors can influence accuracy of alignments if the object is large to require a lot of scans. Laser scanning data has random errors so accuracy of alignments can be improved by matching error distributions of pairs of point clouds to natural distributions. This method is called "best fitting" in contrast "shape matching" in the software, PolyWorks |Inspector. Accuracy of alignments between shape matching and best fitting was discussed by using the scan data of three phase-shift laser scanners (FARO Focus 3D MS120, FARO Focus 3D X330 and $\mathrm{Z}+\mathrm{F}$ Imager 5016) and two time-of-flight scanners (Leica BLK 360 and Leica Scan station C5). As a result of demonstrations, a shape matching method can keep errors in range from millimetres to a few centimetres whereas a best fitting method can keep errors less than a few millimetres according to comparison with distributions of points in reality. Moreover, accuracy of alignments varies between time-of-flight laser and phase-shift laser scanners. Those results are restrictive due to using point clouds in which qualities of each data vary widely. However accuracy of alignments by using shape matching and best fitting methods is demonstrated by showing points with histograms of error distributions.

\section{ACKNOWLEDGEMENTS}

The authors would like to acknowledge Parco Archeologico di Ostia Antica for permitting the investigation.

\section{REFERENCES}

Barber, D. and J. Mills, J. (revised by Mills, J and Andrews, D), 2011. 3D Laser Scanning for Heritage (second edition) Advice and guidance to users on laser scanning in archaeology and architecture, Swindon

Boehler, W., Vicent, M. B., \& Marbs, A. 2003. Investigating laser scanner accuracy. The International Archives of Photogrammetry, Remote Sensing and Spatial Information Sciences, 34(Part 5), 696-701.

Calantropio, A., Chiabrando, F., Rinaudo, F., \& Losè, L. T. 2018. Use and evaluation of a short range small quadcopter and a portableimaging laser for built heritage 3D documentation. International Archives of the Photogrammetry, Remote Sensing \& Spatial Information Sciences, 42(1).

Devrim, A. K. C. A., 2003a. "Full Automatic Registration of Laser Scanner Point Clouds". Optical, 3, Zurich, pp.22-25.

Dold, C., and Brenner, C., 2004a. Automatic matching of terrestrial scan data as a basis for the generation of detailed 3D city models. International Archives of Photogrammetry, Remote Sensing and Spatial Information Sciences, 35(B3), 1091-1096.

Dold, C., and Brenner, C., 2006a. Registration of terrestrial laser scanning data using planar patches and image data. International Archives of Photogrammetry, Remote Sensing and Spatial Information Sciences, 36(5), 78-83.

Fröhlich, C., \& Mettenleiter, M. 2004. Terrestrial laser scanning-new perspectives in 3D surveying. International archives of photogrammetry, remote sensing and spatial information sciences, 36(Part 8), W2.

San José Alonso, J. I., Martínez Rubio, J., Fernández Martín, J. J., \& García Fernández, J. 2011. Comparing time-of-flight and phase-shift. The survey of the royal pantheon in the basilica of San Isidoro (Leon). Int. Archives of Photogrammetry and Remote Sensing and Spatial Information Sciences, 38(5).

Low, K. L. 2004. Linear least-squares optimization for point-toplane icp surface registration. Chapel Hill, University of North Carolina, 4(10).

Makadia, A., Patterson IV, A. and Daniilidis, K., 2006a. Fully automatic registration of 3D point clouds. In Computer Vision and Pattern Recognition, 2006 IEEE Computer Society Conference on (Vol. 1, pp. 1297-1304). IEEE, Philadelphia

Mechelke, K., Kersten, T. P., \& Lindstaedt, M. 2007. Comparative investigations into the accuracy behaviour of the new generation of terrestrial laser scanning systems. Proc. in the Optical, 3, 19-327.

Tian, J., Schneider, T., Straub, C., Kugler, F. and Reinartz, Mechelke, K., Kersten, T. P., \& Lindstaedt, M. 2007. Comparative investigations into the accuracy behaviour of the new generation of terrestrial laser scanning systems. Proc. in the Optical, 3, 19-32 
The International Archives of the Photogrammetry, Remote Sensing and Spatial Information Sciences, Volume XLII-2/W9, 2019 8th Intl. Workshop 3D-ARCH “3D Virtual Reconstruction and Visualization of Complex Architectures", 6-8 February 2019, Bergamo, Italy

Toth, C. K., \& Petrie, G. 2018. Terrestrial laser scanners. In Topographic Laser Ranging and Scanning (pp. 29-88). CRC Press. 